\title{
El principio de contextualidad y relación de los documentos: una aproximación tentativa
}

\author{
Alejandro Delgado Gómez \\ Archivo Municipal de Cartagena (España) \\ 3000 Informática
}

\section{Resumen}

Se realiza una exploración preliminar del concepto de contexto y de los problemas asociados. Para ello, se adopta la perspectiva de un modelo transfinito de contexto. Desde esta perspectiva se aborda el contexto de creación, gestión y conservación de documentos, enfocándose sobre los convencionales contextos jurídicoadministrativo, de procedencia, procedimental, documental y tecnológico. De igual modo, se explora el contexto de uso de los documentos, sobre la base de la teoría archivística postmoderna. Durante esta exploración se discuten los principios de procedencia y de orden original. Por último, se declaran una serie de recomendaciones: la investigación en profundidad, la revisión crítica de nuestras técnicas descriptivas y la sugerencia de un nuevo principio, el de contextualidad y relación, como principio básico en complejos entornos burocráticos y tecnológicos.

Palabras clave: Contexto. Contexto de creación. Contexto de uso. Descripción archivística. Metadatos. Principio de orden original. Principio de procedencia. Transfinito.

\section{Abstract}

A preliminary exploration of the concept of context, as well as its associated problems, is presented. In order to reach this aim, the perspective of a transfinite model of context is adopted. From this perspective, the context of creation, management and preservation of records is analysed, focusing on the conventional juridical-administrative, provenancial, procedural, documentary and technological contexts. Similarly, the context of use of the records is explored, on the basis of post-modern archival theory. During this exploration, principles of provenance and original order are discussed. Finally, the text states a set of recommendations: a deep research on the concept of context, a critical review of our descriptive techniques, and the suggestion of a new principle, the one of contextuality and relationship, as essential principle in complex bureaucratic and technological environments.

Keywords: Archival description. Context. Context of creation. Context of use. Metadata. Principle of original order. Principle of provenance. Transfinite.

Scire. $12: 1$ (en.-jun. 2006) 23-46. ISSN 1135-3761. 


\section{Introducción}

El presente texto se aplica a una exploración preliminar del concepto de contexto de los documentos, esencial para la teoría y la práctica archivística, con el objeto de mostrar el modo en el que se ha problematizado en los actuales entornos burocráticos y tecnológicos, de manera que una aproximación sencilla, como la que ha regido en nuestro modelo archivístico durante décadas, ya no resulta eficaz, ni desde el punto de vista de la dilucidación teórica, ni desde el de la aplicación práctica al diseño y uso de sistemas archivísticos, sean manuales o automatizados. Se define esta exploración como preliminar en la medida en que, aunque se intenta proporcionar garantía literaria e instanciación empírica suficientes para todos los argumentos, esto no ha sido posible en todos los casos, de modo que el presente texto se expone como sujeto a posterior profundización y debate.

Se entiende que el problema del contexto no es exclusivo de la archivística. En los últimos años, teóricos de diferentes disciplinas como las ciencias sociales (Dilley, 1999), la lingüística (Duranti y Goodwin, 1992), la crítica literaria (Culler, 1992), la empresa (Maruyama, 1992), la información (Veltman, 2005b), o la inteligencia artificial (McCarthy, 1996; McCarthy y Buvac, 1998) han abordado desde sus diversas perspectivas el mismo problema, que puede sintetizarse, en la versión más simple, mediante la expresión "nada es auto-explicativo"; y, en la más compleja, "a nada se le puede atribuir un solo significado". En la investigación archivística también puede rastrearse la expresión de estas dos versiones, y de toda una gama intermedia (Nesmith, 1993).

Para abordar adecuadamente el concepto de contexto de los documentos, el presente texto parte de la petición de principio, igualmente provisional, de que el contexto es transfinito, haciendo uso por analogía, o, de manera más estricta, haciendo uso metafórico, de un término procedente de la teoría clásica de conjuntos (Cantor, 2006). No debe entenderse esta petición de principio como un intento de aproximarse al problema del contexto desde un punto de vista matemático, sino, como se ha dicho, simplemente como una metáfora para indicar características del contexto tales como su naturaleza no lineal y no contable, o la existencia de infinitos contextos, en lo que llamaríamos un "continuo de contextualidad", del que cada sistema extrae aquellos elementos discretos que en cada caso resultan relevantes o de utilidad.

A efectos de análisis, se aborda el problema del contexto, en primer lugar, en el sistema del creador/conservador, que en el presente texto tienen igual consideración; en segundo lugar, desde el sistema de uso. Esta distinción, como se ha indicado, es puramente analítica, y no responde al carácter transfinito del contexto, tal y como se entiende en el párrafo anterior. Es decir, ambos sistemas se influyen mutuamente en una relación variable y no predecible, y su exposición secuencial únicamente pretende mejorar la legibilidad. Para mostrar los problemas asociados

Scire. $12: 1$ (en.-jun. 2006) 23-46. ISSN 1135-3761. 
al contexto en el sistema de uso, se utiliza la teoría archivística postmoderna, frecuentemente apoyada en el pensamiento francés postestructuralista (Foucault, 2001; Derrida, 1996) y en la sociología de la estructuración (Giddens, 1995), del documento y el archivo como instrumentos de poder. Para mostrar los problemas asociados al contexto en el sistema del creador/conservador, se hace uso de los cinco contextos identificados por el Proyecto InterPARES 1 (Duranti, 2005). No debe entenderse que el presente texto asuma que solo existen estos cinco contextos. Más bien, son una guía útil a la finalidad del mismo. Para cada uno de los contextos se asume que son complejos, entendiendo con ello que la complejidad crece con la evolución, que genera complejidad, y que crece con la conectividad (Davis, 2005); y se expone una muestra de esta potencial complejidad asociada y de los problemas que la misma podría generar en los sistemas archivísticos. Los contextos de procedencia y documental se tratan de manera parcialmente conjunta.

En el caso de los contextos de procedencia y documental, así como en el caso del contexto procedimental, se han utilizado además para discutir la aplicabilidad sin modificaciones de los convencionales principios archivísticos de procedencia y orden original. No debe entenderse que se argumenta contra estos principios, sino, como se ha dicho, contra su uso sencillo en entornos complejos. El presente texto expone en el apartado de conclusiones una serie de propuestas para contribuir a minimizar los problemas asociados a una consideración simple del contexto de los documentos.

\section{Definición del problema}

Convencionalmente, se define contexto como " 1 . Las circunstancias organizativas, funcionales y operativas que circundan la creación, recepción, almacenamiento, o uso de los materiales, y su relación con otros materiales. 2. Las circunstancias que un usuario puede aportar a un documento y que influyen la comprensión del documento por parte de ese usuario" (Pearce-Moses, 2005). El contexto es, además, también convencionalmente, uno de los tres aspectos que constituyen el documento o, de manera más amplia, un objeto de información. Los otros dos son el contenido, es decir, aquello de lo que trata o que contiene el documento u objeto; y la estructura, es decir, el modo en que se conforma el documento u objeto y el modo en que sus distintos elementos establecen entre ellos una relación más o menos estable, predefinida y previsible (Gilliland-Swetland, 2000a). Por último, se está generalizando la convención de que, en entornos digitales, el contenido viene cada vez con más frecuencia representado en forma de datos, mientras que el contexto y la estructura vienen cada vez con más frecuencia representados en forma de metadatos (McKemmish et al., 1999c).

El presente texto entiende que dentro de la tradición archivística que se construye entre mediados del siglo XIX y mediados del XX, se tiende a pensar que Scire. $12: 1$ (en.-jun. 2006) 23-46. ISSN 1135-3761. 
existe un solo contexto lineal y finito, o un conjunto limitado de contextos también lineales y finitos, y que el archivero puede dar cuenta de ellos con las herramientas analíticas al uso (Thomassen, 1999). Por ejemplo, en la tradición de los archivos considerados históricos, se tiende a identificar el contexto con el contexto de procedencia, que se puede expresar de manera unívoca y sin confusión, mediante la asignación de un fondo a un creador y de un creador a un fondo. En la tradición de la gestión de documentos, se tiende a identificar el contexto con un conjunto limitado de contextos — los procedimientos administrativos, la procedencia o el régimen jurídico-, reductibles también a un modo de expresión único. Esto en lo que se refiere a los aspectos relacionados con la creación, recepción, almacenamiento y en general gestión de los documentos. En lo que se refiere al uso de los documentos, se tiende a pensar que "las circunstancias que un usuario puede aportar a un documento y que influyen su comprensión del documento por parte de ese usuario" son equivalentes a las circunstancias que el archivero ha aportado ya al documento - por regla general, mediante la asignación de la procedencia, y por tanto el contexto de uso del documento también es susceptible de expresión con facilidad (Ketelaar, 2001, 2002).

Por contraste, queremos explorar un conjunto de aserciones que cuestionarían las asunciones mencionadas en el párrafo anterior. Estas aserciones son: 1) que el contexto nunca es lineal y finito, y que no existe nada parecido a un conjunto limitado de contextos (Ketelaar, 2001, 2002); 2) que las circunstancias organizativas, funcionales y operativas en que se crea, gestiona y conserva el documento son más complejas, en las burocracias contemporáneas, de lo que pudiera creerse a simple vista (Trace, 2002); 3) que los marcos de gestión de documentos forman parte de marcos más amplios de sistemas de información, sistemas de conocimiento y, en último extremo, sistemas sociales, que influyen en aquellas circunstancias (Upward, 2001); 4) que los documentos no se relacionan exclusivamente con otros materiales, sino también con funciones, procesos, agentes, e incluso son relaciones de más de un orden; y que tal conjunto de relaciones no está predefinido de una vez por todas (McKemmish, 2001); 5) que un análisis detallado de la procedencia debiera hacer explícito que esta nunca es simple (Hurley, 1995); 6) que, en lo que se refiere al uso, el archivero no puede prever las circunstancias que un usuario aportará al documento en diferentes contextos de recuperación (Ketelaar, 2001, 2002); 7) que, en un entorno tecnológico cambiante, el documento ya no es nunca un objeto simple, sino un objeto terriblemente compuesto y distribuido, y sus aspectos contextuales pueden a menudo, y a menudo sucede, solaparse con sus aspectos estructurales (Gilliland y Eppard, 2000; Duranti y Thibodeau, 2006). Todo ello ha sido puesto de manifiesto por las modernas tecnologías de la información, las tecnologías de convergencia universal y los sistemas de realidad virtual, pero el análisis sigue siendo similar en el caso de documentos que no se están generando en la 
actualidad, es decir, en el caso de documentos que fueron gestionados en el pasado y de los que se considera que han sobrevivido a causa de su valor histórico.

Situémonos en un sistema contemporáneo de creación, gestión y conservación de documentos. Los documentos creados tienen un conjunto de contextos, del que podemos acordar, por convención, que es el conjunto de contextos identificados por el proyecto InterPARES 1 (Duranti, 2005): un contexto jurídico-administrativo, un contexto de procedencia, un contexto procedimental, un contexto documental y un contexto tecnológico. Téngase en cuenta que InterPARES 1 se movía en el marco de la investigación archivística con una fuerte base en la tradición jurídica y una orientación positivista. Es decir, a InterPARES 1 le interesaba sobre todo el modo en que se generaban documentos en los sistemas administrativos. Con independencia de que cada uno de estos contextos se definiera a un nivel de exhaustividad suficiente (MacNeil, 2004), la identificación de los mismos a efectos de la investigación de InterPARES 1 era impecable. Otros tipos de sistemas probablemente requerirían la definición de otros contextos. Por ejemplo, en un archivo personal debieran ser inmediatamente relevantes el contexto social o cultural, el contexto familiar o el psicológico. En un archivo científico debieran ser relevantes el contexto profesional —incluida la deontología profesional— o el de la tradición científica en la que se desenvuelve el archivo. En un archivo de prensa local debieran ser relevantes el contexto cultural inmediato, el político o el lingüístico. Es decir, del hecho de que, en nuestros entornos de trabajo y en nuestras tradiciones de trabajo, operemos con un solo contexto, o con un conjunto limitado de contextos, no se sigue necesariamente que el contexto, o los conjuntos de contextos, sea (n) limitado (s), sino, más bien, que no precisamos mayor articulación para llevar a cabo nuestro trabajo. Lo que cabe preguntar es si en el entorno actual la articulación que ha sido suficiente durante algo más de un siglo sigue siendo suficiente: nos desenvolvemos en un marco de irresponsabilidad política y social graves; esta irresponsabilidad ha traído consigo el requerimiento de creación, gestión y uso de documentos responsables y con auténtico valor evidencial (Piggott y McKemmish, 2002); y estos documentos responsables y evidenciales se generan mediante tecnologías fluctuantes, imprecisas y altamente perecederas (Duranti, 2005; Duranti y Thibodeau, 2006) que, además, contribuyen, mediante la mencionada convergencia universal, a incrementar aquel marco de irresponsabilidad (Veltman, 2005a).

En las siguientes secciones se explorará la posibilidad de obtener algo de luz al respecto, a partir del análisis de los contextos convencionales en un sistema corporativo de creación, gestión y conservación de documentos. El contexto jurídicoadministrativo, en el marco de InterPARES 1, se define como "el sistema legal y organizativo al que pertenece el cuerpo creador". El contexto de procedencia se define como "el cuerpo creador, su mandato, estructura y funciones". El contexto

Scire. $12: 1$ (en.-jun. 2006) 23-46. ISSN 1135-3761. 
procedimental, como "el procedimiento en el curso del cual se crea un documento". El contexto documental, como "el fondo archivístico al que pertenece el documento, y su estructura interna". El tecnológico, como "las características del hardware, el software y otros componentes del sistema informático electrónico en el que se crean los documentos" (Duranti, 2005).

\section{Los contextos de creación, gestión y conservación de los documentos}

Tómese en primer lugar el contexto jurídico-administrativo. Está compuesto por todas las leyes o documentos con rango normativo a los que debe sujetarse una organización a efectos, por lo que nos interesa, de creación, gestión, conservación y uso de documentos. En el caso, digamos, de un ayuntamiento, esto incluye legislación de carácter genérico, como la legislación de procedimiento administrativo, la de régimen local, la legislación sobre grandes ciudades, la legislación sobre firma electrónica y DNI digital, la legislación sobre patrimonio histórico, la de propiedad intelectual, la de protección de datos, etc.; más los reglamentos internos de aplicabilidad general. Además, para cada una de sus áreas — urbanismo, medio ambiente, hacienda, servicios sociales, policía, etc.- existen conjuntos normativos de aplicación específica. En el caso de ciertas competencias, por otra parte, esto sucede a diferentes niveles jurisdiccionales — comunitario, estatal, autonómico y local. Por supuesto, un buen sistema de gestión de documentos debiera dar cuenta de todo este corpus normativo, lo cual no es difícil, en la medida en que, por amplio que sea, no deja de ser finito y, si se ordena de acuerdo con algún criterio, por ejemplo cronológico, también lineal. Sin embargo, un buen sistema de gestión de documentos debiera prever que un corpus legislativo no está nunca terminado. Por ejemplo, un artículo especialmente conflictivo de una ley puede generar una sentencia judicial susceptible de convertirse en jurisprudencia. Si esta sentencia se adecúa a las previsiones del sistema respecto al modo de resolver el conflicto, entonces no sucede nada, pero si la sentencia es desfavorable al sistema, entonces el sistema tendría que modificarse para adecuarse a la jurisprudencia así generada. Un segundo ejemplo: puede que un determinado conjunto de artículos de una ley dada tengan que enmendarse para cumplir con alguna nueva normativa comunitaria. $\mathrm{Si}$ el sistema se ha creado de manera cerrada, bajo la asunción de que la ley no se modificaría, entonces el sistema debe sufrir un proceso de reingeniería. Sin embargo, si el sistema ha previsto ciertos niveles de flexibilidad, entonces solo precisará de ciertos reajustes, quizá la eliminación de algunos valores de atributo en algunos campos de la base de datos y la inclusión de otros valores nuevos. Hace algunos años, la organización para la que trabaja el autor del presente texto intentó desarrollar un sistema de gestión de documentos desde el momento de creación de los mismos. En el caso del área de urbanismo, se diseñó un substancial núme- 
ro de decretos, cuya legislación de base se especificaba en el cuerpo del documento, no mediante, por ejemplo, campos para la selección semiautomática de valores de atributos. Puesto que el proceso de diseño ocupó más de un año, durante este tiempo hubo que modificar varias veces cientos de plantillas de decretos, a medida que se modificaba la ley. El proceso se hubiera mejorado de haber expresado el contexto jurídico mediante campos estructurados con listas de valores fijos.

De estos ejemplos, por otra parte, se desprende que el contexto jurídico-administrativo no es lineal: lo sería si, a partir de una sentencia desfavorable o de una enmienda a una ley, se cerrara el sistema, se migraran los datos y se abriera un nuevo sistema; pero esto no es lo habitual; una modificación del sistema para ajustarse a estos cambios legislativos no sucede "al final", sino en algún punto "a lo largo" del sistema.

Por lo demás, el contexto jurídico-administrativo guarda una estrecha relación, e influye en otros contextos, por ejemplo el procedimental, en la medida en que el procedimiento de, digamos, una concesión de licencia de obras, tiene que acomodarse a la legislación al respecto. Si la legislación especifica que un informe técnico es un requisito preceptivo previo a la resolución, un expediente de concesión de licencia de obras que se resuelva sin este informe técnico no será conforme con la legislación. De igual modo, si la legislación especifica que determinadas actividades medioambientales tienen que ser informadas por una Comunidad Autónoma, esto afectará al contexto procedimental —el procedimiento es más lento y complejo-, y al de procedencia, en la medida en que se incrementa el número de agentes que participan en la creación del expediente. También podría darse el caso inverso: si un determinado procedimiento se muestra ineficaz o perjudicial para los intereses de las organizaciones o, de manera más importante, de la ciudadanía, bien pudiera ser que se modificara la ley, mediante una interminable secuencia de recursos, para modificar el procedimiento.

El contexto de procedencia constituye un reto mucho más complejo: el principio de procedencia se encuentra fuertemente enraizado en nuestra tradición archivística y forma parte del cuerpo central de axiomas que toda ciencia necesita para construir sobre él el conjunto de proposiciones teóricas y de aplicaciones prácticas que le permiten funcionar. De una ciencia puede, por regla general, discutirse todo, salvo el cuerpo central de axiomas, que está "en el límite", formando parte del sistema pero al mismo tiempo fundamentándolo. Desde la conocida perspectiva kuhniana, cuyo concepto de paradigma ha sido adoptado por la archivística postmoderna, este cuerpo central solo puede modificarse por vía de revolución, cuando ha dejado de ser eficaz, ha generado demasiadas complejidades en el sistema y, sobre todo, cuando existe otro cuerpo que puede substituirlo. Es decir, mientras no existe un paradigma alternativo, el sistema genera complejidad, incluso complejidad ineficaz, con el fin de poder seguir funcionando con el paradigma existente

Scire. $12: 1$ (en.-jun. 2006) 23-46. ISSN 1135-3761. 
(Kuhn, 1981). Un segundo modelo explicativo del funcionamiento de la ciencia, no revolucionario, sino, por continuar con la terminología política, reformista, es el falsacionismo refinado propuesto por Imre Lakatos, no tan popular en la archivística postmoderna, de acuerdo con el cual un sistema puede ir sufriendo modificaciones coherentes, sin tocar su cuerpo central, o, de manera más precisa, un paradigma científico siempre aceptará un cierto número de aserciones del paradigma anterior, que contribuyen a su propio enriquecimiento (Lakatos, 2002). La pregunta es ipuede la archivística seguir funcionando con los principios que se construyeron entre mediados del siglo XIX y mediados del siglo XX, debiera modificarlos de manera razonable y coherente, o debiera llevar a cabo el cambio de paradigma que muchos teóricos y practicantes están solicitando desde diferentes ámbitos?

Tal y como han explicado distintos investigadores en los últimos años (Cook, 1997; Gilliland-Swetland, 2000; Thomassen, 1999), nuestro actual paradigma archivístico, apoyado en los principios de procedencia y de orden original, con ligeras variaciones nacionales, lejos de estar constituido por algo así como "leyes de la naturaleza archivística", viene determinado por criterios históricos e ideológicos bien identificados. Por ejemplo, la procedencia y el orden original coexistieron con el principio temático de pertinencia utilizado por los archiveros franceses, hasta que en la primera reunión conjunta de archiveros y bibliotecarios en Bruselas se decidió que los dos primeros satisfacían mejor las necesidades de la Archivística (GillilandSwetland, 2000). Sin embargo, la pertinencia se siguió utilizando en una de las primeras ordenaciones de los Archivos Nacionales de Canadá, ya en el siglo XX (Nesmith, 2002). Por lo demás, las necesidades de la archivística aún eran fuertemente dependientes de la investigación histórica. Recuérdese que Sir Hilary Jenkinson no formuló su principio de respeto al fondo, o de santidad de la evidencia, hasta bien entrado el siglo XX; y Theodore Schellenberg introdujo el modelo gerencial archivístico, apoyado en la discriminación de las edades de los documentos, que ha sobrevivido hasta la actualidad, bastante más tarde (Tschan, 2002). En el actual entorno archivístico, sin embargo, la cuestión no es si la procedencia, o, en sentido estricto, la procedencia única o simple es una ley natural de los archivos o un resultado de fuerzas históricas en competición, sino, más bien, si resulta funcional, útil y operativa para crear, gestionar y conservar los documentos. El principio de procedencia se ha cuestionado sobre la base de diversos argumentos, siendo el que nos interesa a efectos del presente texto el desarrollado por Chris Hurley, a saber, la procedencia es un procedimiento circular de asignación de responsabilidad (Hurley, 1995). De acuerdo con el argumento de Hurley, definiciones de "fondo" y de "creador" como las establecidas por ISAD (G) (1) no dicen nada ni sobre el fondo ni sobre el creador. Si a la pregunta "¿quién es el creador del fondo del Ayuntamiento de Cartagena?" respondemos "el Ayuntamiento de Cartagena", y a la pregunta “¿qué es el Ayuntamiento de Cartagena?” respondemos "el creador del fondo

Scire. $12: 1$ (en.-jun. 2006) 23-46. ISSN 1135-3761. 
del Ayuntamiento de Cartagena", ciertamente no estamos diciendo gran cosa. Este argumento forma parte de una detallada teoría de descripción archivística elaborada por Hurley y enmarcada en la tradición del sistema australiano de serie, que, en filigrana, considera que la procedencia no debiera venir dada por el nombre del órgano que presuntamente creó los documentos, sino por las funciones que dan lugar a que los documentos se creen (Hurley, 1993, 1994, 1995). Esto tiene algunas consecuencias para la teoría de la descripción archivística: por ejemplo, el abandonar el fondo como unidad prioritaria de descripción a favor de la serie —aunque posteriores refinamientos han explicado que en realidad este análisis puede aplicarse a cualquier nivel que tenga sentido para la organización (Bearman, 1996)_; el integrar, lejos de separar como sucede en nuestra tradición, la clasificación, entendida no como cuadro sino como sistema, dentro del proceso de descripción (2); el reconocimiento de la existencia de "funciones ambientales" o sociales más amplias que las que se incorporan en las funciones de una organización (Hurley, 1995); o, de manera substancial, la deconstrucción de nuestro "órgano creador" único en un agregado de agentes que intervienen en el proceso de crear el documento y, más allá, en la deconstrucción de la biografía del órgano creador, separando a efectos analíticos el documento de su contexto de procedencia, para reunificarlos posteriormente mediante relaciones más ricas y complejas (Hurley, 1994).

Continuando con uno de los ejemplos anteriores, de acuerdo con este punto de vista el creador de un expediente de calificación medioambiental no es el Ayuntamiento de Cartagena, sino la función "gestión del medio ambiente", dividida en actividades y tareas, que son responsabilidad del Ayuntamiento y en las que participan varios agentes, cada uno a su vez responsable de una función, actividad o tarea, digamos "emisión de informes jurídicos acerca de las condiciones ambientales", "emisión de informes técnicos con el mismo fin", "redacción de documentos", "autenticación de los documentos redactados", etc. Por supuesto, ya que la función "gestión del medio ambiente" se relaciona con otras del Ayuntamiento, también intervendrán los agentes que ejecutan estas funciones, por ejemplo, la Policía, que desempeña la función de "emitir informes in situ", o el departamento de Hacienda, que desempeña la función de "cobro de los derechos que le corresponden al Ayuntamiento por emitir licencias". Dependiendo además de la legislación, pueden intervenir otros agentes con otras funciones, como la Comunidad Autónoma. Evidentemente, interviene un agente especialmente importante, el interesado, que ejecuta acciones del tipo de "gestionar un disco-pub", su representante, u otras partes interesadas, por ejemplo, el agente "vecino", que desempeña la actividad "sentirse molesto por el ruido del disco-pub". Todo ello sucede, de acuerdo con la propuesta de Hurley, en el marco de funciones más amplias ambientales, por ejemplo la actual preocupación global por protección del entorno, que determinan en

Scire. $12: 1$ (en.-jun. 2006) 23-46. ISSN 1135-3761. 
alto grado el modo en que las funciones anteriores se llevarán a cabo, así como los documentos que generarán.

Pero, como hemos adelantado, esta problematización del contexto no sucede solo en un espacio dado, de manera sincrónica, y en un solo punto del tiempo, sino también a lo largo del tiempo. Hurley utiliza el ejemplo de un matrimonio Habsburgo (Hurley, 1994), nosotros el de una organización con una vida centenaria, como un ayuntamiento. Un cuadro de clasificación elaborado de acuerdo con nuestra tradición incluiría bajo la entrada "función de gobierno" tanto los documentos de gobierno más recientes como aquellos que se generaron, digamos, en 1245. Sin embargo, ¿qué tiene de similar el ayuntamiento que existía en 1245 con el que existe ahora? Durante el Antiguo Régimen, la situación de los ayuntamientos fue razonablemente estable (aunque, por ejemplo, dependiendo de los ayuntamientos, estos podían atravesar por fases más o menos largas de realengo o señorío, que modificaban, de iure o de facto, sus funciones). Los últimos Austrias comienzan a introducir modificaciones sobre las capacidades de los ayuntamientos; pero, de manera especial, la agitada vida política de comienzos del siglo XIX implica muchas y rápidas alteraciones del gobierno municipal (aparición y desaparición de juntas, creación y eliminación de ayuntamientos efímeros, etc.) (Tornel, 2001, 2004). A mediados del XIX la situación se estabiliza, digamos (con lagunas históricas achacables al responsable del presente texto), hasta el estatuto de Primo de Rivera, que vuelve a cambiar la situación. Durante el franquismo la situación vuelve a cambiar, y, una vez más, con el advenimiento de la democracia, alcanzando un cierto grado de estabilidad que, en este momento, con la aplicación de la legislación de grandes ciudades, sufrirá un golpe en determinados municipios. Por lo demás, sería sorprendente encontrar municipios que, desde la Edad Media, hubieran conservado las características que la legislación de bases de régimen local reconoce como definitorias: la misma organización, el mismo territorio geográfico y, mucho menos, la misma población. Naturalmente, aun en el supuesto de que a lo largo de todo este período un ayuntamiento hubiera conservado sus funciones intactas, los órganos y los procedimientos para ejecutar esas funciones se han modificado; es decir, la relación de la organización con sus funciones varía, como varía, por lo demás, la relación de la organización con otras organizaciones. En estas condiciones, ¿qué queremos decir cuando afirmamos que el creador del fondo del Ayuntamiento de Cartagena es el Ayuntamiento de Cartagena? Una persona, en cierto sentido, no es nunca la misma que era hace veinticinco años; pero, en último extremo, si se requiere que se identifique como la misma persona que fue, se puede acudir a expedientes del tipo de "tener el mismo código genético". En el caso de un ayuntamiento, no existe un código genético que lo identifique como siendo ahora el mismo que era en 1245. El concepto "Ayuntamiento de Cartagena" es solo una abstracción que ha resultado útil durante la fase de infancia, o de elaboración y consoli- 
dación de teoría de la archivística, pero en el complejo entorno actual una lectura también compleja de la procedencia no parece inoportuna.

En esta lectura, el contexto de procedencia de los documentos ciertamente se complica, pero también deviene mucho más rico y útil a efectos de identificación de la responsabilidad, autenticidad, diseño de sistemas, identificación del documento, acceso a y recuperación del mismo, etc. Nótese que en esta lectura no se cuestiona el principio de procedencia mismo, que es extremadamente útil a efectos archivísticos, sino el modo simple y poco informativo en que se ha venido articulando hasta ahora.

En lo que se refiere al contexto procedimental, este delinea el procedimiento mediante el que se crea un documento. Mantiene relación, por ejemplo, con el contexto jurídico-administrativo, en la medida en que el procedimiento genérico, así como muchos procedimientos específicos, vienen dados por ley. Por otra parte, el procedimiento viene ejecutado por un cierto número de agentes que cumplen determinadas funciones, de acuerdo con un modelo complejo de procedencia. A los efectos del presente texto, esto implica que "el procedimiento" no es nunca el procedimiento oficial, tal y como viene dado por ley y/o por especificaciones internas. Además de este procedimiento oficial, que vendría a coincidir más o menos con el que se ve en un diagrama de flujo, o en un flujo de tareas, en el proceso de llevar a término un documento intervienen un cierto número de factores no escritos, muchas veces dependientes del conocimiento tácito de los agentes, y que no quedan reflejados en aquel diagrama de flujo. Por supuesto, mediante técnicas convencionales de diagrama de flujo o de flujo de tareas, o más sofisticadas, como el diseño de ontologías, o, yendo un paso más adelante, mediante técnicas emergentes como la aplicación de la etnografía a entornos burocráticos, estos factores se harían lo suficientemente explícitos como para quedar incorporados, hasta donde sea posible, al procedimiento. En aquellos puntos en los que no sea posible incorporarlos, al menos debiera ser posible minimizar, maximizar o de algún otro modo controlar sus efectos (3). Y, si ni siquiera esto fuera posible, entonces aún debiéramos ser lo suficientemente conscientes de su existencia como para mantener el sistema en funcionamiento, a pesar de la ocurrencia de tales factores. Esto es especialmente evidente en entornos de ejecución de tareas altamente descentralizados. Las organizaciones burocráticas contemporáneas mantienen bajo control gran parte de lo que sucede en cada uno de los PC de la organización; pero no todo lo que sucede, siendo muy ilustrativo el caso de la emisión y recepción de correos electrónicos, que puede que no solo tengan influencia sobre el procedimiento, sino también sobre procesos como la copia de seguridad, el control del spam o la evaluación y selección de documentos.

Por utilizar un segundo ejemplo, una licencia de obras, o de cualquier otro tipo, se concede o se deniega de acuerdo con un procedimiento reglado, consistente, en Scire. $12: 1$ (en.-jun. 2006) 23-46. ISSN 1135-3761. 
el marco de la administración pública, en las fases de iniciación, ordenación, instrucción (con etapas de prueba, informes y participación) y finalización (mediante resolución, por desistimiento o renuncia, o por caducidad). Cada fase se articula, dependiendo del tipo de documento, de una u otra manera, pero básicamente este sería el procedimiento. Sin embargo, además de este procedimiento, para que el documento llegue a su fin intervienen procedimientos del estilo de tipo de procesador de textos utilizado, uso de plantillas para acelerar la redacción, revisión de las primeras versiones de los documentos por parte de la persona jurídica competente, manera de marcar las correcciones que deben hacerse, plazos (que no coinciden con los plazos legales) para marcar estas correcciones y modificar el documento, método de remisión de la solicitud de un informe a otra unidad, método de remisión de ese informe, estilo - dentro de los límites marcados por la burocracia- de redacción de la resolución, manera de atender al interesado durante el período de alegaciones o de audiencia, tipo de relaciones entre los participantes en una tira de cuerda, decisión de acumular expedientes homogéneos, decisión acerca de qué datos se incluirán en el caso de expedientes colectivos, decisión acerca de la manera de cumplimentar los impresos de certificados para enviar las notificaciones a los interesados, decisión acerca del proceso de solicitar a otra unidad el cobro del correspondiente impuesto, etc. Por supuesto, nos referimos al territorio conocido de la elaboración de un expediente; la elaboración de un documento que no fuera un expediente implicaría otra serie de factores de dilucidación más compleja.

A modo de ejemplo, durante el diseño de sistemas mencionado anteriormente para un área de urbanismo, se tomó en consideración todo lo que dijo la gestión de alto nivel, compuesta básicamente por abogados y arquitectos, de la citada área; pero no se entrevistó al personal de primera línea, ni siquiera se visitaron las dependencias de atención al público, de tal manera que, cuando se implantó el sistema, no se había definido ningún proceso para automatizar el envío por correo de las notificaciones a los interesados, ni, por ejemplo, la remisión única y automática de los impuestos a la unidad de Hacienda, con la consecuencia de que el impuesto no se recaudaba o se le recaudaba varias veces a la misma persona. Si se hubieran tenido en cuenta los procesos no escritos, si se hubiera atendido a la fuente de conocimiento tácito que constituyen los auxiliares administrativos, si se hubiera hecho trabajo de campo en las oficinas de primera línea, se hubiera evitado también el colapso del área de urbanismo y la posterior retirada del sistema. Avanzando aún un paso, durante el procedimiento intervienen factores procedentes de un entorno cultural y social más amplio, es decir, tanto el marco social genérico como el marco cultural corporativo en el que se desenvuelven los agentes influyen en el modo en que estos llevan a cabo sus funciones y las reflejan en documentos (Morash, 1984; Trace, 2002; Gracy, 2005; Shankar, 2005). Aunque queda fuera del análisis del contexto procedimental, añadiríamos que, por supuesto, en este llevar a 
cabo sus funciones y reflejarlas en documentos intervienen también factores psicológicos, familiares y, en general, personales, de los que los agentes no son conscientes, que no quedan, ni porque deban ni porque sea posible, reflejados en el diseño del sistema, pero que afectan al mismo en la forma de potenciales excepciones. Una excepción es una situación que el sistema no es capaz de predecir de manera específica, en la medida en que todas las posibles excepciones no se pueden incluir en el análisis y diseño de un sistema, pero cuya ocurrencia genérica sí se puede prever en ese análisis y ese diseño, de tal manera que el sistema debiera incluir un proceso para gestionar las excepciones, sean estas de la naturaleza que sean.

En realidad, esta discusión acerca del contexto procedimental quiere llegar a dos conclusiones diferentes, aunque relacionadas. En primer lugar, en entornos complejos como los de las burocracias contemporáneas, la o las secuencias de pasos que tienen como consecuencia la generación de un documento rara vez son un algoritmo perfecto, una máquina de Turing. Además de los pasos que quedan reflejados en el algoritmo, en el diagrama de flujo, intervienen otros muchos pasos que se han ido aquilatando de manera tácita con el paso de los años, y que no figuran explícitamente en el "procedimiento". Además, intervienen factores ambientales que difícilmente son previsibles, en la medida en que difícilmente se hacen patentes, pero que tienen una profunda influencia en ese procedimiento. A la hora de diseñar un sistema, debiera minimizarse la importancia del procedimiento, y concentrarse en el análisis de los procesos, tanto los oficiales como los tácitos (Cook, 2001). Estos procesos, en muchos casos, podrán analizarse, hasta cierto nivel de exhaustividad, mediante técnicas de diagrama de flujo o de flujo de tareas; pero el conocimiento tácito difícilmente se hará explícito sin el recurso a técnicas como la del diseño de ontologías. Más allá de esto, los factores ambientales externos nunca saldrán a la luz si no se utilizan métodos similares a los que utiliza la investigación etnográfica. La propuesta del presente texto en lo que se refiere al contexto procedimental adopta una aproximación concéntrica. Debiera considerarse el procedimiento oficial como en el centro de un conjunto de circunferencias; los procesos no oficiales, como una circunferencia que engloba el procedimiento, y los factores ambientales como una circunferencia aún mayor que constituye el límite externo.

La segunda conclusión se refiere a otro de los principios fundamentales de la archivística, el de orden original, que es una derivación del Registraturprinzip de origen prusiano. El orden original se mantiene identificando, mediante una secuencia de registros, los pasos ordenados de un procedimiento y los documentos que generan estos pasos. Pero, si en la generación de un documento intervienen pasos que no quedan registrados, e incluso excepciones o factores ambientales que no pertenecen a ninguna secuencia dada, sino que pueden ejercer su influencia en cualquier punto, entonces el orden original es una abstracción cómoda, similar a la abstracción de la procedencia. No obstante, no es en el momento de crear los doScire. $12: 1$ (en.-jun. 2006) 23-46. ISSN 1135-3761. 
cumentos cuando el principio de orden original queda más dañado. Se retomará este asunto en sección posterior, al mencionar la pluralidad de significados que pueden adquirir los documentos en el momento de la recuperación y uso. Nótese que este argumento no dice nada contra el principio de orden original, sino contra su simplificación. Al igual que sucedía con el principio de procedencia, somos de la opinión de que de la problematización del principio de orden original debiera derivarse una comprensión más rica de la naturaleza del documento y de su gestión, que beneficiaría al modo en que desempeñamos nuestra profesión.

En lo que hace al contexto documental, creemos que el concepto de fondo ha quedado suficientemente discutido al hacer referencia al contexto de procedencia. Sin embargo, si se adopta una visión compleja de la procedencia, y se deshace el vínculo presuntamente necesario entre el fondo y la procedencia, el contexto documental requeriría una posterior investigación, de la que, quizá, no estaría ausente el método de investigación basado en la diplomática archivística contemporánea (MacNeil, 2004).

El contexto tecnológico es quizá el más problemático, no solo por su propia complejidad en el entorno actual, sino por la manera tan absoluta en que las nuevas tecnologías determinan todos los aspectos, tanto de nuestro trabajo como de nuestra vida cotidiana. Todos los documentos han tenido siempre, por supuesto, un contexto tecnológico - la tecnología del minio y la del pergamino, la tecnología de la imprenta, la tecnología de la escritura cortesana...-; pero ninguno de ellos ha sido tan determinante para toda la humanidad como las actuales tecnologías de convergencia universal y el aún emergente desarrollo de sistemas de realidad virtual. Los documentos siempre se han creado con la asistencia de una tecnología que al mismo tiempo determinaba el límite, el modo en que se podían crear los documentos; este límite, en la actualidad, no solo es cada vez más flexible y permisivo, sino, en una lectura detenida y paradójica, absolutamente "limitador". Tratar todos los problemas asociados a la tecnología de creación de documentos en el entorno actual excede la extensión de un solo artículo. Algunos de estos problemas han sido bien estudiados y denunciados. Por ejemplo, el modo en que podrían conservarse a largo plazo documentos creados con un hardware y un software cuya esperanza de vida es mucho más corta que la de los documentos a conservar; o el modo de seguir garantizando la autenticidad de un documento autenticado mediante procedimientos de firma electrónica que no pueden conservarse. Bien que de manera algo arbitraria, se exploran en filigrana dos de las cuestiones tecnológicas relacionadas de modo íntimo con el contexto de los documentos.

En primer lugar, los documentos se están creando y utilizando en entornos crecientemente distribuidos y, en esa medida, difícilmente controlables (Duranti y Thibodeau, 2006; Reed, 1999a, 1999b; Gilliland-Swetland y Eppard, 2000; Davis, 2005). Esto significa, por una parte, que los documentos mismos han pasado a ser 
objetos distribuidos, u objetos asimilables a documentos, formados por componentes que se encuentran en diferentes equipos de una red, o en diferentes equipos de múltiples redes, y que se representan cada vez que se instancian. En estas condiciones, ¿cuál es la identidad del documento? Por ejemplo, una autoliquidación o el pago con tarjeta de crédito en unos grandes almacenes son procesos que implican la interacción entre al menos dos equipos, un cliente y un servidor. Probablemente este servidor remita a su vez a componentes almacenados en otros equipos del sistema, y probablemente entre el cliente y el servidor intervengan otros actores. En cada uno de estos equipos se almacenan datos, no en la forma de documentos tradicionales que reflejan transacciones también tradicionales, sino como componentes parciales de ese objeto asimilable al documento que es el proceso mismo, de tal manera que con la información disponible en un solo equipo no se puede reproducir ni el proceso ni el objeto asimilable a un documento. Por supuesto, esto se complica si intervienen firmas electrónicas generadas mediante infraestructuras de clave pública, que son ellas mismas un proceso de custodia permanente; o si intervienen schemas o DTD alojados en servidores remotos y a los que simplemente se hace referencia en, digamos, una hoja de estilo XSLT. La naturaleza del documento, en estas condiciones de creación, gestión y mantenimiento, es precaria, y los mecanismos para identificar el contexto de creación, gestión y conservación debieran devenir tan precisos como fuera posible, con el fin de evitar la acumulación de documentos, u objetos asimilables a documentos, sin ningún valor, ni como evidencia ni como memoria, bien porque no se puedan reproducir, bien porque no se pueda garantizar su autenticidad una vez reproducidos. Estos mecanismos, por regla general, debieran venir expresados como metadatos normalizados y reutilizables en entornos muy diversos. Tal es la aproximación emprendida, por ejemplo, por el proyecto Clever Recordkeeping Metadata, conocido significativamente como "create once, use many times" (Evans et al., 2006).

En entornos muy distribuidos, disponer de documentos estables es prácticamente imposible, de manera que habría que tender a proporcionar estabilidad mediante la asignación de metadatos. Por lo demás, estos metadatos deberían tener a su vez un alto grado de estabilidad, aunque sus marcos de uso difieran. Si para proporcionar estabilidad al documento se utiliza una serie de metadatos durante la creación y para determinados propósitos, otra serie durante la gestión y para otros propósitos, otra durante la conservación, etc., entonces solo se está añadiendo inestabilidad a lo inestable. Por el contrario, si desde el momento de la creación, o incluso antes, se pudieran definir las necesidades previstas de gestión, conservación y uso de los documentos, se podría iniciar una secuencia coherente de asignación de metadatos, que pasarían junto con los datos por todas las etapas de gestión y uso que atraviesa un documento, contribuyendo a dotarlo de identidad y, en esa misma medida, de estabilidad.

Scire. $12: 1$ (en.-jun. 2006) 23-46. ISSN 1135-3761. 
Tan interesante como el problema de la estabilidad de los documentos resulta la tendencia (Nesmith, 2002; Hurley, 2004) de las organizaciones, o, en su caso, de las personas, a generar documentos, $\mathrm{u}$ objetos asimilables a documentos, de manera independiente de las normas y buenas prácticas recomendadas. Es decir, a pesar de los empeños realizados en los últimos años para proponer mejores métodos de creación, gestión y conservación de documentos, los documentos se crean, se gestionan y se conservan sin atender a estas propuestas: por ejemplo, haciendo uso de software propietario, sin definición de mecanismos de copia de seguridad, sin una adecuada política de asignación de metadatos, etc. Como se ha dicho, los sistemas de gestión de documentos forman parte de o están relacionados con sistemas más amplios de gestión de la información, de gestión del conocimiento o de gestión de datos; los documentos son información, aportan conocimiento y están conformados por datos, pero no pueden tratarse, tal y como se derivaría de algunas propuestas recientes, como si fueran meramente información, conocimiento o datos (Hurley, 2004). Por supuesto, esta es una vía de menor resistencia, permite un tratamiento integrado y plantea menos problemas, en la medida en que obvia muchos de ellos. Sin embargo, los documentos son información, conocimiento y datos que tienen que cumplir unos requisitos específicos, si es que tienen que servir como evidencia y memoria de algo. El hecho de que tengan que cumplir con estos requisitos no significa que no sean integrables con sistemas más amplios de información, conocimiento y datos; de hecho, es deseable que esta integración tenga lugar, y, yendo más allá, que todos estos sistemas se integren a su vez en los sistemas de procesos de la organización, como una manera de minimizar el riesgo asociado al alejamiento de las buenas prácticas. Es decir, no se puede pedir a una organización que mantenga un sistema de gestión de procesos, otro de gestión de la información, otro de gestión de documentos, otro de gestión del conocimiento, etc. Por esta vía, las organizaciones se desviarán hacia la línea de menor esfuerzo y atenderán a sus necesidades inmediatas, no a las necesidades sociales más amplias de creación, gestión y conservación de buenos documentos a efectos de evidencia y memoria. No obstante, que los sistemas se integren no significa necesariamente que se deba renunciar a satisfacer los requisitos propuestos por las normas y buenas prácticas, sino, más bien, que los responsables de que estos requisitos se cumplan deben intervenir de manera más activa en el diseño de tales sistemas integrados.

\section{El contexto desde el punto de vista del uso de los documentos}

En los últimos años han sido varios los autores que han hecho notar el modo en que el uso de los documentos está mediado por sistemas implícitos o explícitos de poder que sitúan al creador/conservador en una posición de privilegio con respecto al usuario último (Piggott y McKemmish, 2002; Cook y Schwartz, 2002a, 2002b; Ketelaar, 2001, 2002; Harris, 2002; O'Toole, 2002), de tal manera que los 
contextos de creación influyen en el contexto de uso. Esto es especialmente cierto en los modelos dictatoriales, en los que, junto a la compulsiva necesidad de generar documentos que permitan mantener el control sobre los ciudadanos, existe una necesidad igualmente compulsiva de no mostrar estos documentos. Pero también sucede en los modelos democráticos, incluidos aquellos que disponen de leyes explícitas de libertad de información y acceso. Un ejemplo muy evidente de ejercicio de poder es la redacción misma de los documentos. Sin entrar en los detalles del cuerpo informativo de, por ejemplo, un decreto, simplemente los encabezados del tipo "antecedentes de hecho" y "fundamentos de derecho", en lugar de encabezados del tipo "esto fue lo que sucedió" y "esto es lo que dice la ley con respecto a lo que sucedió", ya suponen una limitación en el contexto de uso, procedente de los contextos jurídico-administrativo y procedimental anteriormente examinados.

Mucho más interesante resulta, desde el punto de vista archivístico, el rol implícito de los archiveros como sacerdotes del templo de los documentos (Ketelaar, 2002). En efecto, los archiveros tienden a mostrarse como jugando un humilde papel secundario (Nesmith, 2002), pero lo cierto es que, por ejemplo, las normativas de acceso a las salas de investigación y, una vez en ellas, a los documentos físicos, constituyen, sobre la base de que los documentos tienen necesidades precisas de conservación, una herramienta de poder que deja en manos del archivero la decisión acerca de quién accede, quién no y bajo qué condiciones. Por supuesto, los documentos deben conservarse, pero el actual contexto tecnológico, que, por una parte, es fuente de generación de problemas, se erige también en este caso en instrumento facilitador, en la medida en que, por ejemplo, anula el argumento de las necesidades de conservación de los documentos mediante procesos de digitalización e incorporación a la web. Para el documento, esto implica el alejamiento del contexto en que se creó, gestionó y conservó, y la adquisición de nuevos significados, no controlables por el archivero, cada vez que un usuario remoto lo reactiva. Para el archivero, implica la pérdida del control sobre el significado del documento y, por tanto, la pérdida de su rol como "hermeneuta" exclusivo del templo archivístico. Por ejemplo, es posible que un archivo tienda a creer, de manera intuitiva, que ciertas áreas temáticas interesan más al conjunto de los usuarios y que, por tanto, deben describirse con mayor detalle. Estas áreas incluyen convencionalmente en, digamos, un archivo municipal, la historia del urbanismo y las hidalguías. Sin embargo, catas tentativas realizadas con software cuantitativo (como DADiSP SE 2002) y cualitativo (como NVivo 7) podrían sugerir que los intereses de los usuarios están mucho más diversificados, que el interés sobre la historia del urbanismo se concentra en determinados períodos — probablemente aquellos en los que los estudiantes de arquitectura tienen que realizar trabajos de clase-, y que el interés en los estudios de población no se enfoca sobre el estudio genealógico, sino en motivaciones mucho más prácticas, para las que no se requieren los ex-

Scire. $12: 1$ (en.-jun. 2006) 23-46. ISSN 1135-3761. 
pedientes de hidalguía. Si los resultados de estas catas se probaran, los archiveros de este archivo estarían ejerciendo un abuso de poder sobre las descripciones y el acceso a los documentos, a partir del supuesto de que sabemos lo que el usuario quiere sin posterior comprobación.

A cambio de la pérdida de poder mencionada, se mejoraría la accesibilidad al documento y su democratización, así como el enriquecimiento de su contexto en una red de documentos susceptibles de reorganizarse de infinitas maneras, de establecer infinitas relaciones y de adoptar, en consecuencia, infinitos significados (McKemmish, 2005). Principios como el de procedencia y el de orden original quedan por esta vía extremadamente relativizados, y técnicas archivísticas como la de la descripción deben reconsiderarse, en función de estos infinitos usos del documento.

\section{Recomendaciones}

El presente texto es únicamente una aproximación inicial al problema del contexto, y, como toda aproximación inicial, tiene lagunas, puntos débiles y argumentados de manera pobre, y cierto nivel de provisionalidad. Nuestra primera recomendación se enfoca sobre la necesidad de una exploración en profundidad del concepto de contexto y de los problemas asociados al mismo. Herramientas metodológicas como el análisis conceptual y el trabajo etnográfico de campo, así como el posterior análisis de sus datos, debieran resultar imprescindibles a este respecto. De igual modo, una exploración del concepto de contexto y sus problemas asociados en entornos web cada vez más generalizados, y en el marco de las tecnologías de convergencia universal y de los emergentes sistemas de realidad virtual, merecería atención más detallada (Veltman, 2005a, 2005b; Reed, 1999a, 1999b; Iacovino, 1999).

En segundo lugar, gran parte de los problemas que se han mencionado a lo largo del presente texto se minimizarían, o al menos podrían abordarse con mayor eficacia, si se reconsiderara la técnica, fundamental a la práctica archivística, de la descripción. La descripción no debiera tener lugar cuando los documentos han terminado su ciclo de vida y han pasado a constituir un fondo, sino desde el momento de la creación de los mismos, o, más allá, antes de la existencia de estos, en el momento de diseñar los sistemas de creación y gestión. Como se indicó al comienzo, el contexto viene representado mediante metadatos, y una adecuada política de asignación de metadatos automatizados hasta donde sea posible y fuertemente normalizados contribuiría a mantener la identidad de los documentos y la representación de su contexto con diferentes finalidades. Los archiveros tendemos a creer que una descripción a nivel de ítem no es practicable, habida cuenta de los enormes volúmenes de información que manejamos, y, bien que de manera inconsciente, tendemos a mostrar un cierto menosprecio por los bibliotecarios, que manejan aparentemente volúmenes mucho menores. Estas afirmaciones, a falta de contraste posterior, nunca han sido ciertas, pero lo son mucho menos en entornos 
distribuidos altamente complejos. En la actualidad no existe límite para los volúmenes de información que podrían manejar los bibliotecarios, y la biblioteconomía es una disciplina que tiene mucho que enseñarnos, a efectos de descripción de grandes masas de documentos. A diferencia de la práctica archivística de la "gran descripción", apoyada en una cantidad mínima de metadatos estructurados y enormes porciones de texto narrativo que dificultan la identificación inmediata de elementos dados de información, los bibliotecarios han desarrollado tradicionalmente lenguajes de descripción altamente estructurados que permiten manejar de manera homogénea y casi rutinaria grandes masas de información. Los archiveros se han resistido a esta estructuración de los lenguajes descriptivos sobre la base de que cada archivo es un mundo y no se pueden hacer compatibles los lenguajes necesarios en cada uno de ellos. Aproximaciones custodiales como ISAD (G) y EAD (Encoded Archival Description) han mostrado que esto no es necesariamente cierto, en la medida en que, si bien los documentos procedentes de cada organización o persona son exclusivos de esa organización o persona, tales organizaciones y personas tienden a ser más rutinarias de lo que los archiveros quisieran. Todos los documentos de un ayuntamiento, de un laboratorio científico o de una cadena de televisión serán razonablemente similares a los de otros ayuntamientos, laboratorios científicos o cadenas de televisión; y en sus metadatos figurarán probablemente un identificador codificado, un identificador textual, uno o varios agentes, diversas fechas, etc. Por esta vía, es concebible argumentar una aproximación altamente estructurada a la descripción archivística como técnica de asignación de metadatos capaces de vérselas con contextos de creación, gestión, conservación y uso complejos (McKemmish et al., 1998a, 1998b, 1999a, 1999b, 1999c, 1999d, 1999e; Evans et al., 2006; Gilliland et al., 2005).

Finalmente, el presente texto quisiera sugerir un desplazamiento de paradigma que no implique la sustitución del paradigma positivista por el posmoderno, sino más bien la adopción de un paradigma posmoderno que conserve lo mejor del positivista. Esta sugerencia toma forma en la definición de un principio de primer orden, en el que quedarían englobados, como principios de segundo orden, los de procecencia y orden original. Este principio, al que el autor del presente texto llama tentativamente principio de contextualidad y relación de los documentos, se articularía, provisionalmente, en los siguientes términos: "los documentos tienen un contexto de creación, gestión y conservación complejo, y son susceptibles de relaciones múltiples. Es función del archivero identificar y dilucidar tanto el contexto de creación, gestión y conservación como las relaciones que se producen en estas fases. Los documentos también tienen un contexto de uso complejo e impredecible, y son susceptibles de adquirir durante los procesos de uso relaciones igualmente complejas, múltiples e impredecibles. Es función del archivero sentar las bases —mediante aquella identificación y dilucidación del contexto de creación, gestión

Scire. $12: 1$ (en.-jun. 2006) 23-46. ISSN 1135-3761. 
y conservación, y de las relaciones que se producen en estas fases- para que los documentos puedan utilizarse en diferentes contextos, establecer diferentes relaciones y adoptar diferentes significados. Todo ello se consigue mediante la asignación de metadatos que preceden a la existencia del documento, le acompañan durante toda su vida y perduran una vez que el documento se ha extinguido".

Sería deseo del autor del presente texto que el principio provisional enunciado moviera a un mayor debate en la tradición archivística en lengua hispana.

\section{Notas}

(1) Creador es el órgano corporativo, familia o persona que creó, acumuló y/o mantuvo los documentos en el curso de la actividad personal o corporativa. Fond es el total de documentos, con independencia de la forma o soportes, creados y/o acumulados y utilizados por una persona particular, familia u órgano corporativo en el curso de las actividades y funciones de ese creador (ISAD [G], 2000).

(2) Los proyectos del Records Continuum Research Group SPIRT - Recordkeeping Metadata Schema, convertido en norma, y el actualmente en curso Clever Recordkeeping Metadata son ejemplos de aproximación global a la teoría de la descripción y la asignación de metadatos.

(3) La excelente norma australiana AS 5090 prevé todas las variaciones posibles a los procesos de trabajo, pero no las excepciones, es decir, todo aquello que el sistema no recoge ni puede predecir, pero que debería tener capacidad para gestionar, si sucediera.

\section{Referencias}

Bearman, David (1996). Item Level Control and Electronic Recordkeeping. // Archives \& Museum Informatics. 10:3 (1996) 195-245. URL: <http://www.archimuse.com/ papers/nhprc/item-lvl.html>. Consulta: 2006-08-27.

Cantor, Georg (2006). Fundamentos para una teoría general de conjuntos: escritos y correspondencia selecta. Barcelona: Crítica, 2006.

Cook, Terry (2001). Archival science and postmodernism: new formulations for old Concepts. // Archival Science. 1 (2001) 3-24.

Cook, Terry (1997). What is Past is Prologue: A History of Archival Ideas Since 1898, and the Future Paradigm Shift. // Archivaria. 43 (Spring 1997) 17-63. URL: <http://www.mybest docs.com/cookt-pastprologue-ar43fnl.htm>. Consulta: 2006-08-27.

Cook, Terry; Schwartz, Joan (2002a). Archives, Records, and Power: From (Postmodern) Theory to (Archival) Performance. //Archival Science. 2:3-4 (September 2002) 171-185.

Cook, Terry; Schwartz, Joan (2002b). Archives, Records, and Power: The Making of Modern Memory. // Archival Science. 2:1-2 (March 2002) 1-19.

Culler, Jonathan (1992). Sobre la deconstrucción. Madrid: Cátedra, 1992.

Davis, Ben Howell (2005). Cumulative complexity: Understanding the scope of digital asset liquidity, continuity, and viability. // Journal of Digital Asset Management. 1:1 (2005) $16-24$. 
Derrida, Jacques (1996). Mal de archivo: una impresión freudiana. Madrid: Trotta, 1996. Dilley, Roy (ed.) (1999). The Problem of Context. Nueva York: Berghahn, 1999.

Duff, Wendy; Harris, Verne (2002). Stories and Names: Archival Description as Narrating Records and Constructing Meanings. // Archival Science. 2:3-4 (Sept. 2002) 263-285.

Duranti, Alessandro; Goodwin, Charles (eds.) (1992). Rethinking Context: Language as an Interactive Phenomenon. Cambridge: Cambridge University Press, 1992.

Duranti, Luciana (ed.) (2005). La conservación a largo plazo de documentos electrónicos auténticos: hallazgos del proyecto InterPARES. Cartagena: Ayuntamiento; 3000 Informática, 2005.

Duranti, Luciana; Thibodeau, Kenneth (2006). The Concept of Record in Interactive, Experiential and Dynamic Environments: the View of InterPARES. Inédito; pendiente de publicación en Archival Science.

Evans, Joanne; McKemmish, Sue; Bhoday, Karuna (2005). Create Once, Use Many Times: The Clever Use of Recordkeeping Metadata for Multiple Archival Purposes. // Archival Science. 5 (2005) 17-42.

Foucault, Michel (2001). La arqueología del saber. México: Siglo XXI, 2001.

Giddens, Antohny (1995). La constitución de la sociedad: bases para la teoría de la estructuración. Buenos Aires: Amorrortu, 1995.

Gilliland, Anne J. (2000a). Setting the Stage. // Introduction to Metadata: Pathways to Digital Information. Los Ángeles: The Getty Institute, 2000. URL: <http://www.getty.edu/ research/ conducting_research/standards/intrometadata/setting.html>. Consulta: 200608-27.

Gilliland-Swetland, Anne (2000b). Enduring Paradigm, New Opportunities: The Value of the Archival Perspective in the Digital Environment. Washington DC: Council on Library and Information Resources, 2000. URL: <http://www.clir.org/pubs/reports/ pub89/pub89.pdf>. Consulta: 2006-08-27.

Gilliland-Swetland, Anne J. y Eppard, Philip B. (2000). Preserving the Authenticity of Contingent Digital Objects. // D-Lib Magazine 6: 7-8 (July-August 2000). URL: <http://www.dlib.org/dlib/july00/eppard/07eppard.html>. Consulta: 2006-08-27.

Gillilan, Anne, Rouche, Nadav, Lindberg, Lori, Evans, Joanne (2006). Towards a 21st Century Metadata Infrastructure Supporting the Creation, Preservation and Use of Trustworthy Records: Developing the InterPARES 2 Metadata Schema Registry. // Archival Science. 5 (2005) 43-78.

Gracy, Karen F. (2004). Documenting Communities of Practice: Making the Case for Archival Ethnography. // Archival Science. 4 (2004) 335-365.

Harris, Verne (2002). The Archival Sliver: Power, Memory, and Archives in South Africa. // Archival Science. 2:1-2 (March 2002) 63-86.

Hurley, Chris (1993). What if anything, is a function? // Archives and Manuscripts. 2. 21 (1993). URL: <http://www.sims.monash.edu.au/research/rcrg/publications/whatif.html>. Consulta: 2006-08-27.

Hurley, Chris (1994). The Australian ('Series') System: An Exposition. // The Records Continuum: Ian Maclean and Australian Archives first fifty years. Clayton: Ancora Press,

Scire. $12: 1$ (en.-jun. 2006) 23-46. ISSN 1135-3761. 
1994. URL: <http://www.sims.monash.edu.au/research/rcrg/publications/chtrc1.html>. Consulta: 2006-08-27.

Hurley, Chris (1995a). Ambient Functions - Abandoned Children to Zoos. // Archivaria. 40 (Fall 1995). URL: <http://www.sims.monash.edu.au/research/rcrg/publications/ ambientf.htm>. Consulta: 2006-08-37.

Hurley, Chris (1995b). Problems with Provenance. // Archives and Manuscripts. 23:2 (1995). URL: <http://www.sims.monash.edu.au/research/rcrg/publications/provenance.html>. Consulta: 2006-08-27.

Hurley, Chris (2000). The Making and Keeping of Records: (2) The Tyranny of Listing. // Archives and Manuscripts. 28:1 (May 2000). URL: <http://www.sims.monash.edu.au/ research/rcrg/publications/makingkeeping2.html>. Consulta: 2006-08-27.

Hurley, Chris (2004). What, If Anything, Is Records Management? // RMAA Conference, Canberra, September 2004. URL: <http://www.sims.monash.edu.au/research/rcrg/ publications/ch-what.pdf>. Consulta: 2006-08-27.

Hurley, Chris (2006a). Relationships in Records: (1) What, If Anything, Is a Relationship? // Records Continuum Research Group Publications. URL: <http://www.sims.monash. edu.au/research/rcrg/publications/relations1.htm>. Consulta: 2006-08-27.

Hurley, Chris (2006b). Relationships in Records: (2) How Do I Own Thee? Let Me Count the Ways. // Records Continuum Research Group Publications. URL: <http://www.sims. monash.edu.au/research/rcrg/publications/relations2.htm>. Consulta: 2006-08-27.

Iacovino, Livia (1999). Regulatory, Recordkeeping and Legal Issues associated with Managing Websites. // Managing Websites Seminar, November-December 1999. Australian Society of Archivists, 1999. URL: <http://www.archivists.org.au/sem/papers.html>. Consulta: 2006-08-27.

ISAD (G) (2000). General International Standard Archival Description. Second Edition. Ottawa: International Council on Archives, 2000.

Ketelaar, Eric (2001). Tacit Narratives: The Meanings of Archives. // Archival Science. 1 (2001) 131-141.

Ketelaar, Eric (2002). Archival Temples, Archival Prisons: Modes of Power and Protection. // Archival Science. 2 (2002) 221-238.

Kuhn, Thomas S. (1981). La estructura de las revoluciones científicas. México: Fondo de Cultura Económica, 1981.

Lakatos, Imre (2002). La metodología de los programas de investigación científica. Madrid: Alianza, 2002.

MacNeil, Heather (2004). Contemporary Archival Diplomatics as a Method of Inquiry: Lessons Learned from Two Research Projects. // Archival Science. 4 (2004) 199-232.

Maruyama, Magoroh, ed. (1992). Context and Complexity: Cultivating Contextual Understanding. New York: Springer, 1992.

McCarthy, John (1996). A logical AI aprroach to context. // John McCarthy's Home Page, 1996. URL: <http://www-formal.stanford.edu/jmc/index.html>. Consulta: 2006-08-27.

McCarthy, John; Buvac, Sasa (1998). Formalizing context: Expanded notes. // John McCarthy's Home Page, 1998. URL: <http://www-formal.stanford.edu/jmc/index.html>. Consulta: 2006-08-27. 
McKemmish, Sue (2001). Placing Records Continuum Theory and Practice. //Archival Science. 1 (2001) 333-359.

McKemmish, Sue; Acland, Glenda (1999a). Accessing Essential Evidence on the Web: Towards an Australian Recordkeeping Metadata Standard. // The Web after a Decade: Proceedings of AusWeb99, the Fifth Australian World Wide Web Conference, April 1999. URL: <http://ausweb.scu.edu.au/aw99/papers/mckemmish/>. Consulta: 2006-08-27.

McKemmish, Sue; Acland, Glenda; Cumming, Kate (1999b). The End of the Beginning: The SPIRT Recordkeeping Metadata Project. // Archives at Risk: Accountability, Vulnerability and Credibility, 1999 Annual Conference of the Australian Society of Archivists, July 1999. URL: <http://www.sims.monash.edu.au/research/rcrg/publications/ asaq99.html>. Consulta: 2006-08-27.

McKemmish, Sue; Acland, Glenda; Cumming, Kate; Reed, Barbara; Ward, Nigel (1999c). Recordkeeping Metadata Project. Glossary. Monash: Records Continuum Research Group, 1999. URL: <http://www.sims.monash.edu.au/research/rcrg/research/spirt/ glossary.html>. Consulta: 2006-08-27.

McKemmish, Sue; Acland, Glenda; Reed, Barbara (1999d). Documenting Business: The Australian Recordkeeping Metadata Schema. // Paper for ADCS'99, Australasian Document Computing Symposium, December 1999. URL: <http://www.sims.monash. edu.au/research/rcrg/publications/adcs.html>. Consulta: 2006-08-27.

McKemmish, Sue; Acland, Glenda; Reed, Barbara (1999e). Towards a Framework for Standardising Recordkeeping Metadata: The Australian Recordkeeping Metadata Schema. // Records Management Journal. 9:3 (December 1999). URL: <http://www.sims.monash. edu.au/research/rcrg/publications/framewrk.html. Consulta: 2006-08-27.

McKemmish, Sue; Acland, Glenda; Ward, Nigel, Reed, Barbara (1999f). Describing Records in Context in the Continuum: the Australian Recordkeeping Metadata Schema. // Archivaria. 48 (Fall 1999). URL: <http://www.sims.monash.edu.au/research/rcrg/ publications/archiv01.htm>. Consulta: 2006-08-27.

McKemmish, Sue; Cunningham, Adrian; Parer, Dagmar (1998a). Metadata Mania: Use of Metadata for Electronic Recordkeeping and Online Resource Discovery. // Place, Interface and Cyberspace: Archives at the Edge, Proceedings of the 1998 Conference of the Australian Society of Archivists, Fremantle 6-8 August 1998. URL: $<$ http://www.sims.monash.edu.au/research/rcrg/publications/recordkeepingmetadata/ sm01.html>. (Consulta: 2006-08-27).

McKemmish, Sue, Parer, Dagmar (1998b). Towards Frameworks for Standardising Recordkeeping Metadata. // Archives and Manuscripts. 26:1 (1998). URL: $<$ http://www.sims.monash.edu.au/research/rcrg/publications/recordkeepingmetadata/ smckrmp1.html>. Consulta: 2006-08-27.

Morash, Merry (1984). Establishment of a Juvenile Police Record: The Influence of Individual and Peer Group Characteristics. // Criminology. 22 (February 1984) 97-111.

Nesmith, Tom (ed.) (1993). Canadian Archival Studies and the Rediscovery of Provenance. Metuchen: Scarecrow Press, 1993.

Nesmith, Tom (2002). Seeing Archives: Postmodernism and the Changing Intellectual Place of Archives. The American Archivist. 1:65 (Spring-Summer 2002) 24-41.

Scire. $12: 1$ (en.-jun. 2006) 23-46. ISSN 1135-3761. 
O’Toole, James M. (2002). Cortes's notary: The symbolic power of records. // Archival Science. 2:1-2 (March 2002) 45-61.

Pearce-Moses, Richard (2005). A Glossary of Archival and Records Terminology. Chicago: Society of American Archivists, 2005. URL: <http://www.archivists.org/glossary/ index.asp>. Consulta: 2006-08-27.

Piggott, Michael; McKemmish, Sue (2002). Recordkeeping, Reconciliation and Political Reality. // Australian Society of Archivists Annual Conference, Sydney, August, 2002. URL: <http://www.sims.monash.edu.au/research/rcrg/publications/piggottmckemmish 2002.pdf>. Consulta: 2006-08-27.

Reed, Barbara (1999a). Archives of the New Millenium: Exploring the archival issues of the early twenty-first century. // 23rd Annual Conference of Archives and Records Association of New Zealand, Auckland, July 1999. URL: <http://www.sims. monash.edu.au/ research/rcrg/publications/brep2a.html>. Consulta: 2006-08-27.

Reed, Barbara (1999b). Legal and recordkeeping issues associated with management of websites. // Managing Websites Seminar, November-December 1999. Australian Society of Archivists, 1999. URL: <http://www.archivists.org.au/sem/papers.html>. (Consulta: 2006-08-27).

Shankar, Kalpana (2004). Recordkeeping in the Production of Scientific Knowledge: An Ethnographic Study. // Archival Science. 4 (2004) 367-382.

Thomassen, Theo (1999). The development of archival science and its European dimension. // Arkivarien och Arkivvetenskapen. Seminarium för Anna Christina Ulfsparre/ The Archivist and the Archival Science. Seminar for Anna Christina Ulfsparre. Lund: Swedish National Archives, 1999. 75-83. URL: <http://www.archiefschool.nl/docs/thomdeve.pdf>. Consulta: 2006-08-27.

Tornel Cobacho, Cayetano (2001). El gobierno de Cartagena en el Antiguo Régimen: 12451812. Cartagena: Ayuntamiento, 2001.

Tornel Cobacho, Cayetano (2004). Gobierno local y quiebra del Antiguo Régimen en Cartagena: antecedentes (1808-1812). Murcia: Academia Alfonso X el Sabio, 2004.

Trace, Ciaran B. (2002). What is Recorded is Never Simply 'What Happened': Record Keeping in Modern Organizational Culture. // Archival Science. 2 (2002) 137-159.

Tschan, Reto (2002). A Comparison of Jenkinson and Schellenberg on Appraisal. // The American Archivist. 2:65 (Fall-Winter, 2002) 176-195.

Upward, Frank (2001). Modelling the continuum as paradigm shift in recordkeeping and archiving processes, and beyond - a personal reflection. // Records Management Journal (Nov 2001). URL: <http://www.sims.monash.edu.au/research/rcrg/publications/ Frank\%20U\%20RMJ\%202001.pdf> Consulta. 2006-08-27.

Veltman, Kim H. (2005a). Retos para las aplicaciones de TIC/TCU en el patrimonio cultural. // Patrimonio cultural y tecnologías de la información: a la búsqueda de nuevas fronteras. Cartagena: Ayuntamiento; 3000 Informática, 2005. 13-51.

Veltman, Kim H. (2005b). Understanding new media: augmented knowledge and culture. Calgary: University of Calgary Press, 2005. URL: <http://www.sims.monash.edu.au/ research/rcrg/publications/Frank> U RMJ 2001.pdf. Consulta: 2006-08-27. 